\title{
NUMERICAL PREDICTION OF SPRINGBACK IN SHEET METAL FORMING IN ANISOTROPIC ELASTOPLASTIC FINITE STRAINS
}

\author{
Díaz R. ${ }^{1 *}$ and Nguyen K. ${ }^{1}$ and Montáns F.J. ${ }^{1}$ and Sanz M.A. ${ }^{1}$ \\ ${ }^{1}$ Universidad Politécnica de Madrid, Escuela Técnica Superior de Ingeniería Aeronáutica y del Espacio, \\ Departamento de Aeronaves y Vehículos Espaciales, Plaza Cardenal Cisneros 3, 28040 Madrid, Spain \\ raul.diaz.gonzalez@alumnos.upm.es, khanhnguyen.gia@upm.es, fco.montans@upm.es, \\ miguelangel.sanz@upm.es
}

Key words: Springback, Sheet Metal Forming, Anisotropy, Plasticity, Nonlinear, Contact Problems

\begin{abstract}
We present an application on springback of an anisotropic elastoplastic finite strain model. The computational procedure allows the sole use of internal elastic variables as it is fully hyperelastic. Based on the Lee-type multiplicative decomposition, which permits an additive solution in a logarithmic strain approach, it resolves the "rate issue" by the employment of a corrector rate of elastic strains and its algorithmic implementation [1]. The associative nature and Clausius-Duhem inequality recover the formulation of Simo's isotropic strain-hardening particular case. Its application to metal and soft materials has been corroborated as it is also valid for anisotropic yield functions and for any anisotropic stored energy (linear and nonlinear in logarithmic strains) [2]. The computation of the stress-point algorithm is done using a simple backward-Euler scheme. Its validation has been performed by the implementation of the procedure into a subroutine which allows the user to compute benchmark models in the commercial program ADINA [3]. This permits to explore its usefulness in application for the numerical prediction of springback in thin sheet metal forming processes. The results are compared with both experimental and other authors' results. Moreover, many simulations were performed such as the draw-bending test, the unconstrained bending problem, the square cup or the 3DS warping benchmark.
\end{abstract}

\section{INTRODUCTION}

The springback phenomenon is a undesirable change in the final shape of metal forming manufactured parts when the unloading follows the plastic forming process. This effect has great dependence on the elastoplastic material properties and the plastic evolution on strain hardening. The geometry is also another important aspect if large displacements are present.

The first issue to successfully simulate springback is related to the constitutive model considered, specially plastic hardening, which is responsible for the residual stress resultant in the forming process. Sheet metal parts exhibit anisotropic plastic behaviour due to the texture, a property dependent on the metallic grain morphology, distribution and orientation. During the plastic forming, when large deformations take place, this anisotropy causes important effects over the final shape, being also sensitive to the residual stress distribution.

The objective of this paper is to show the applicability, efficiency and robustness of the novel framework 
$[1,2]$ to simulate and predict a complex springback benchmark. Also, we describe in a brief manner the formulation used in this paper, implemented as a user subroutine in the commercial finite element program ADINA $^{\circledR}$. After that, we demonstrate the applicability to the prediction of the springback phenomenon.

\section{CONTINUUM THEORY AND ALGORITHMIC FRAMEWORK IN THE ELASTOPLAS- TIC MODEL}

In this section we show the most important aspects of the computational framework, from the continuum theory to the algorithmic framework employed to the stress calculation.

The elastoplastic material model is motivated from the rheological model shown in Fig. 1. This macroscopic model may be described in infinitesimal strains, where $\varepsilon$ and $\sigma$ are the measurable variables, and later permit us an easy extension to the respective finite variables $E$ and $T$ to define the elastoplastic behaviour in finite strains, commonly referred as large strains.

The device is composed of two branches. The first one is a Prandtl-type branch which includes an elastic Hookean spring in a series disposition with a plastic dissipative friction element. It is in this part where the internal state variables simultaneously operate: elastic strain and plastic strain. The second branch, placed in parallel disposition, only contains a Hookean spring element, being responsible for the macroscopic kinematic hardening, where only the external variable total strain operates.
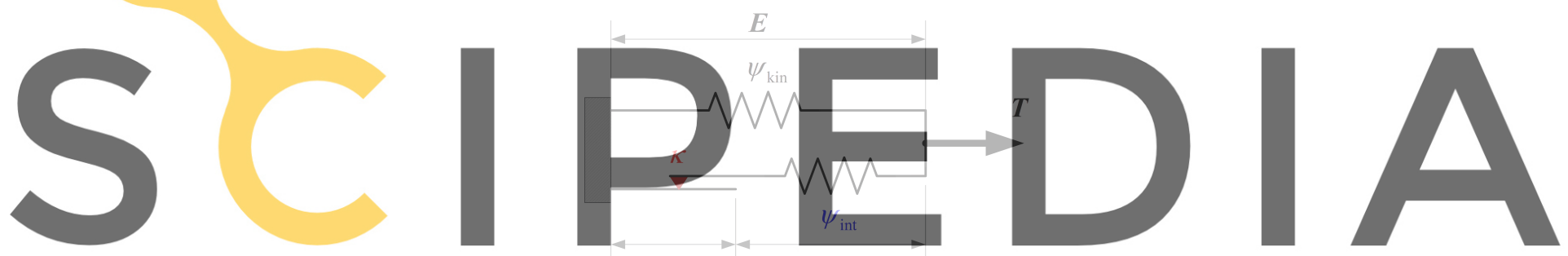

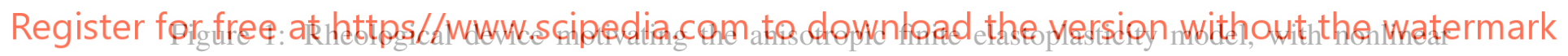
hardening

The model employs the Lee-type multiplicative decomposition over the deformation gradient $F$, split into an elastic part $F_{e}$ and a plastic part $F_{p}$ (Eq.1), as shown in Fig. 1.

$$
F=F_{e} F_{p}
$$

We are interested in the use of logarithmic strains. This strain measure permits us to describe the logarithmic tensor strain variables: $E_{e}=\frac{1}{2} \ln \left(F_{e}^{T} F_{e}\right)$ and $E=\frac{1}{2} \ln \left(F^{T} F\right)$, and obtain the internal state dependence $E_{e}\left(E, F_{p}\right)$. This operation is important for this work, because it will permit us to get the strain rate tensor into the addition of two contributions in the incremental nonlinear continuum theory

$$
\dot{E}_{e}\left(\dot{E}, \dot{F}_{p}\right)=\left.\frac{\partial E_{e}}{\partial E}\right|_{\dot{F}_{p}=0}: \dot{E}+\left.\frac{\partial E_{e}}{\partial F_{p}}\right|_{\dot{E}=0}: \dot{F}_{p}=\left.\dot{E}_{e}\right|_{\dot{F}_{p}=0}+\left.\dot{E}_{e}\right|_{\dot{E}=0}
$$


where the first term is obtained within a continuum framework, when the dissipative plastic strain in rate form is frozen; whereas the second term is obtained when the total strain rate remains frozen. This condition occurs when evolution is formulated in the incremental framework, where events occur sequentially and incrementally. This split additive operation is the Wilkins corrector-predictor scheme, and Eq. 2 is then formulated as

$$
\dot{E}_{e}\left(\dot{E}, \dot{F}_{p}\right)=\left.\dot{E}_{e}\right|_{\dot{F}_{p}=0}+\left.\dot{E}_{e}\right|_{\dot{E}=0}={ }^{t r} \dot{E}_{e}+{ }^{c t} \dot{E}_{e}
$$

where ${ }^{t r} \dot{E}_{e}$ is the predictor, or trial elastic contribution to the elastic logarithmic strain rate tensor $\dot{E}_{e}$ with a frozen plastic evolution $\left(\dot{F}_{p}=0\right)$. The second term is the corrector, the plastic contribution to the elastic logarithmic strain rate tensor $\dot{E}_{e}$, where the external variable total strain rate $\dot{E}$ remains frozen.

\subsection{Dissipation and elastic logarithmic strain rate}

The stored energy function may be written in terms of any elastic strain measure. We use the logarithmic strains for convenience, as related in the rheological model (see Fig. 1), the elastic energy function is written in terms of logarithmic strains as

$$
\Psi\left(E, E_{e}\right)=\Psi_{k i n}(E)+\Psi_{\text {int }}\left(E_{e}\right)
$$

where $\Psi_{k i n}(E)$ is the elastic strain energy associated to the variable total strain, which operates in the "external" spring element, and $\Psi_{\text {int }}\left(E_{e}\right)$ is the internal energy function associated to the elastic strain which operates in the "internal" spring element. The stored energy $\Psi$ can be split into a volumetric hyperelastic part and a deviatoric elastoplastic part. Energy contributions are formulated in quadratic form, i.e.

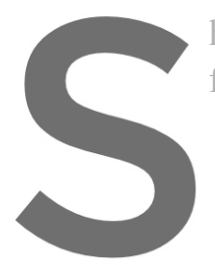

$\mu_{k i n}$ and $\mu_{i n t}$ are the elastic material shear moduli (Lame's constant) of the parts (internal and external)

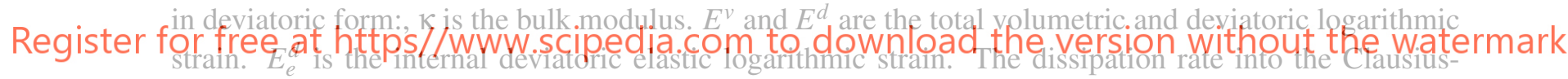

Dunhem inequality is written as

$$
\mathcal{D}=\mathcal{P}-\dot{\Psi}=\mathcal{P}-\dot{\Psi}_{k i n}-\dot{\Psi}_{\text {int }}
$$

Now, we can define two stress tensors as work-conjugated of the logarithmic strain variables

$$
T_{k i n}=\frac{d \Psi_{k i n}(E)}{d E} \quad \text { and } \quad T_{i n t}^{l e}=\frac{d \Psi_{i n t}\left(E_{e}\right)}{d E_{e}}=\frac{d \Psi_{i n t}\left(E_{e}\right)}{d E_{e}}:\left.\frac{\partial E_{e}}{\partial E}\right|_{\dot{F}_{p}=0}
$$

so the total stress tensor

$$
T=T_{k i n}+T_{i n t}^{\mid e}:\left.\frac{\partial E_{e}}{\partial E}\right|_{\dot{F}_{p}=0}=T_{k i n}+T_{i n t}
$$

The stress tensor $T_{k i n}$ lies in the reference configuration while $T_{i n t}^{\mid e}$ lies in the intermediate configuration as $E_{e}$. The stress $T$ is work-conjugated of the logarithmic strain. Both stresses in Eq. 7 are not expressed in the same finite strain configuration, because $T_{i n t}^{\mid e}$ is described in the intermediate configuration by the 
elastic predictor. Taking into account Eq. 6, in the case of the elastic strain rate, with total strain rate frozen, the plastic term is not null $\left(\dot{F}_{p} \neq 0\right)$, and it may be rewritten to the new form

$$
\mathcal{D} \equiv-\left.\dot{\Psi}_{\text {int }}\right|_{\dot{E}=0}=-T_{\text {int }}^{\mid e}:{ }^{c t} \dot{E}_{e}>0 \quad \text { if } \quad \dot{F}_{p} \neq 0
$$

where dissipation is written in terms of a purely elastic strain tensor in the intermediate configuration. The term ${ }^{c t} \dot{E}_{e}$ is named as the elastic corrector strain rate $\dot{E}_{e}$ in logarithmic form. Now, dissipative evolution may be written with a rate of elastic strains $\dot{E}_{e}$, without the use of plastic rates, using an associative flow rule as follows

$$
{ }^{c t} \dot{E}_{e}=-\dot{\gamma} \frac{1}{\frac{2}{3} k} \nabla \phi_{T}=-\dot{\gamma} \frac{1}{\frac{2}{3} k} \mathbb{N}_{T}: T_{i n t}^{\mid e}
$$

where $\mathbb{N}_{T}$ is the Hill anisotropic material parameters fourth order tensor, used to introduce anisotropic plastic deformation. Eq. 10 introduces the anisotropic behavior into the evolution law, $\gamma$ is the associative plastic multiplier equivalent to the accumulated plastic strain, $k$ is the yield stress material parameter, and $\phi_{T}$ is plastic potential for the tensor stresses, who drives the elastic corrector step in the incremental algorithm. The yield function may be written with the stress tensor in the intermediate configuration

$$
f_{T}\left(T_{\text {int }}^{\mid e}, k\right)=T_{\text {int }}^{\mid e}: \mathbb{N}_{T}: T_{\text {int }}^{\mid e}-\frac{2}{3} k^{2}=0 \quad \text { if } \quad \dot{\gamma}>0
$$

Eq. 10 preserves the classical return mapping schemes of the infinitesimal theory, now with the presence of an orthotropic elastoplastic material and

description in the comput

2.2 Stress integration

The stress integration algorithm is applied over Eq.
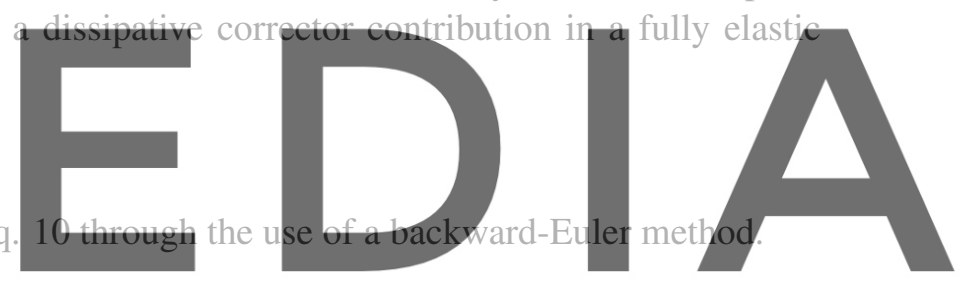

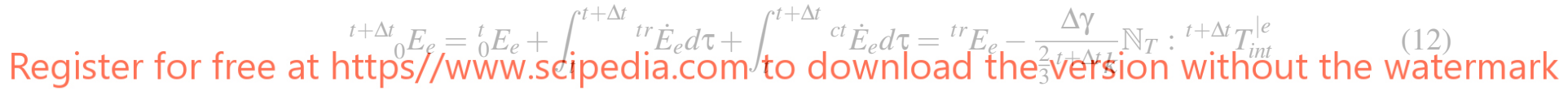

computing the residual as

$$
{ }^{t+\Delta t} \rho_{e}={ }^{t+\Delta t}{ }_{0} \mathbf{E}_{e}+\frac{\Delta \gamma}{\frac{2}{3}^{t+\Delta t} k}{ }^{t+\Delta t} \nabla \phi_{T}-{ }^{t r} \mathbf{E}_{e} \longrightarrow \mathbf{0}
$$

A Newton-Raphson algorithm is used to obtain the correct solution, using the residual ${ }^{t+\Delta t} \mathrm{R}^{(j)} \longrightarrow \mathrm{O}$; $(j)$ represents the local iteration counter from the initial time $t$ to the final time $t+\Delta t$

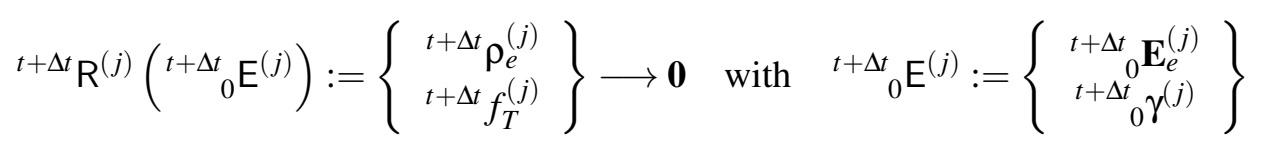

while the Jacobian matrix is

$$
\nabla^{t+\Delta t} \mathrm{R}=\left[\begin{array}{cc}
\frac{d^{t+\Delta t} \rho_{\text {int }}}{d^{t+\Delta t} E_{e}} & \frac{d^{t+\Delta t} \rho_{\text {int }}}{d \Delta \gamma} \\
\frac{d^{t+\Delta t} f_{T}}{d^{t+\Delta t}{ }_{0} E_{e}} & \frac{d^{t+\Delta t} f_{T}}{d \Delta \gamma}
\end{array}\right]
$$


Once the solution has converged, we calculate ${ }^{t+\Delta t} E_{e}$ and ${ }^{t+\Delta t} \gamma$, and we can obtain the internal stresses ${ }^{t+\Delta t} \mathbf{T}_{i n t}^{e}$ in the updated intermediate configuration. The next step is the obtaining of the elastoplastic tangent modulus, with exactly the same form who exhibits the infinitesimal strains case.

\subsection{Computational scheme}

Common finite element programs work with a total lagrangian formulation for large displacements, using the second Piola-Kirchhoff stress tensor and the Green-Lagrange strain tensor. As we use logarithmic strains and their work-conjugated stress, it is required a translation of these internal variables into the stress tensor variables used by finite elements programs, using this consistent algorithmic operation

$$
\begin{gathered}
{ }^{t+\Delta t} \mathbf{S}_{\text {int }}={ }_{0}^{t} \mathbf{X}_{p}^{-1}\left({ }^{t+\Delta t} \mathbf{T}_{\text {int }}^{\mid t r}: \frac{d^{t r} \mathbf{E}_{e}}{d^{t r} \mathbf{A}_{e}}\right){ }_{0}^{t} \mathbf{X}_{p}^{-T} \\
{ }^{t+\Delta t} S={ }^{t+\Delta t} S_{k i n}+{ }^{t+\Delta t} S_{i n t}
\end{gathered}
$$

with the second Piola-Kirchhoff stress tensor ${ }^{t+\Delta t} S$ applied in each element integration point. This term is necessary to update the geometrical stiffness matrix in the nonlinear finite element code. Another necessary term is the elastoplastic tangent modulus, obtained through the mapping operation performed in Eq. 16. This term is mathematically complex, and its details are out of the scope of this paper. We obtain the algorithmic consistent elastoplastic modulus in the form

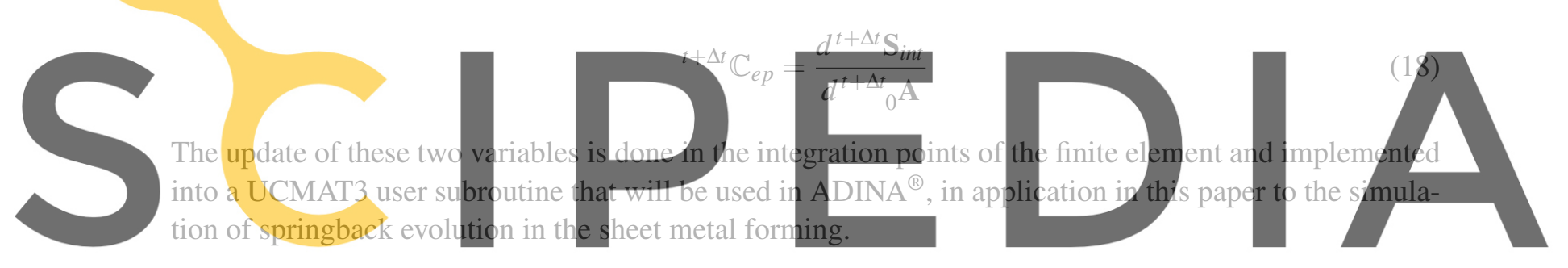

Register för free at https//WWW.SCIPedia.com to download the version without the watermark

In this section, we apply the continuum theory addressed in the previous sections in order to obtain successful predictions of springback in sheet metal formed parts. It is important to put this application into context, as springback simulations are yet not so accurate for their application into industry, where fits or surface roughness are some of these FEM software limitations. In this process of springback prediction, we firstly achieve a fine calibration of the elasto-plastic parameters of the material model, to then use these calibrated materials within the L-bending test and the draw-bending test.

\subsection{CALIBRATION OF ELASTO-PLASTIC PARAMETERS}

The selected materials to be calibrated are TRIP600 (transformation induced plasticity) and DP600 (dual phase) steels; first-generation high-strength steels, widely used in automotive, marine and naval industries, where a compromise solution between low density, great formability and high strength is crucial.

It has been proven [5] that the fitting of the elasto-plastic hardening parameters with the only use of uniaxial tensile tests is not sufficient to accomplish a good calibration of the combined hardening material model when applied to springback prediction. This leads to the need of conducting cyclic experiments such as the Bauschinger test, associated to enormous difficulties in testing conditions. Today's state of art 
is lacking of these cyclic-test experimental data, forcing to find alternative calibration methods. In this work, the calibration of the material parameters is performed through uniaxial tensile test experiments following calibrations in finite element method simulations.

The calibration process starts off with the fitting of the elasto-plastic parameters in Table 1 by using the least mean squares method. This permits the obtaining of $k_{\infty}, \bar{H}^{1}$ and $\delta$ of Eq. 19 which is a Voce-type nonlinear isotropic hardening law. Kinematic hardening is assumed and directly obtained through the decomposition of the shear moduli into both internal $\left(\mu_{i n t}\right)$ and kinematic $\left(\mu_{k i n}\right)$ parts. Previously, the calibration process had determined $k_{0}, \mu_{i n t}$ and $\mu_{k i n}$ through the theory addressed in [2] where the use of the elastic parameters $E$ and $v$ was required. On the other hand, Hill's anisotropy parameters are standardized. This standardization described in [6] is attained through a Gauss-Seidel iterative method with respect the first uni-dimensional stress.

$$
k=k_{0}+\bar{H} \gamma+\left(k_{\infty}-k_{0}\right)\left(1-e^{-\delta \gamma}\right)
$$

Material parameters for TRIP600 and DP600 employed steels are presented in Table 2. This calibration is subsequently checked in a simple numerical Bauschinger test, resulting in curve-fitted graphs such as the one presented in Fig. 2. This color convention will be later re-used: red for experimental data, fuchsia for isotropic, royalblue for kinematic and black for combined hardening. While Materials \#104 and \#107 represent TRIP600 with respectively isotropic and combined hardening; Materials \#172, \#173 and \#174 represent DP600 with isotropic, kinematic and combined material approaches. Note parameters

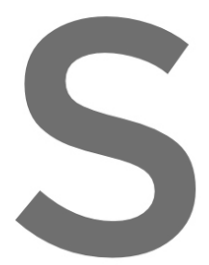
set to zero for both Materi
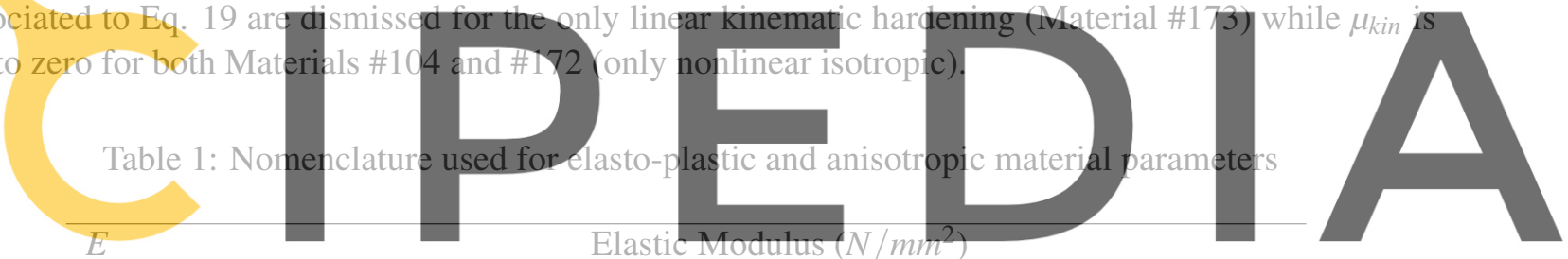

V

Poisson's Ratio (-)

Register for free at https//www.scipedia.cometopedewalogether W/emsion without the watermark

\begin{tabular}{ll}
$k_{\infty}$ & Limit Stress Parameter $\left(\mathrm{N} / \mathrm{mm}^{2}\right)$ \\
$H$ & Linear Hardening Modulus $\left(\mathrm{N} / \mathrm{mm}^{2}\right)$ \\
$\beta$ & Mixed Hardening Parameter ${ }^{a}(-)$ \\
$\bar{H}$ & Effective $^{b}$ Linear Hardening Modulus $\left(\mathrm{N} / \mathrm{mm}^{2}\right)$ \\
$\delta$ & Voce Hardening Parameter $(-)^{2}$ \\
$a, b, c, f, g, h$ & Hill's standardized anisotropy parameters \\
$2 \mu_{\text {int }}(-)$ \\
$2 \mu_{\text {kin }}$ & Internal Shear Moduli $\left(\mathrm{N} / \mathrm{mm}^{2}\right)$ \\
\hline
\end{tabular}

\subsection{FEM MODELING AND SIMULATION RESULTS}

In this section, we will present the results of the simulations carried out in the commercial finite element software ADINA ${ }^{\circledR}$. The prediction of springback geometries is first applied to the L-bending test. The draw-bending test is then approached to re-validate the material model theory in [2], having several

\footnotetext{
${ }^{1} \bar{H}=\beta H$; where $\beta=1$ for isotropic hardening, $\beta=0$ for kinematic hardening. See [3].
} 
Table 2: Calibrated material parameters

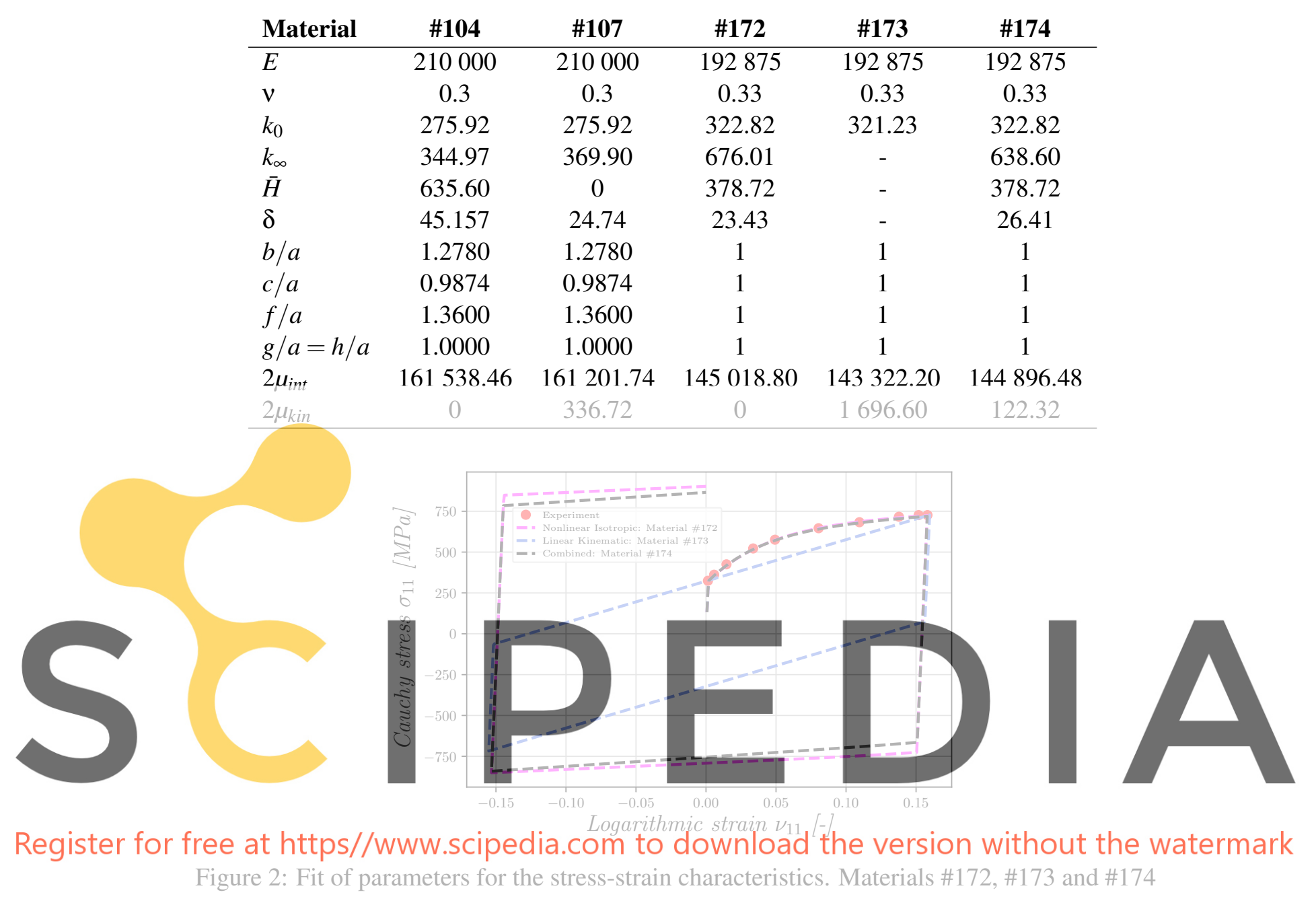

similarities to [7], obtaining more precise results. All simulations presented in this work are computed in an implicit static analysis scheme.

\subsubsection{L-BENDING TEST}

The geometrical tooling layout of this test is presented in Fig. 3, where various dimensions are given. Dimensions and experimental data have been taken from [4]. The metallic sheet is placed between a blank holder and a die that will clamp the specimens during the bending process which spans the whole punch stroke. Above the cantilever part of the specimen, a punch $\left(R_{p}=3 \mathrm{~mm}\right)$ is initially off-contact. The dimensions of the specimens are $61.2 \times 11.3 \times 0.8 \mathrm{~mm}$. The die radius is set to $R_{d}=3 \mathrm{~mm}$ while the gap distance between the punch and the die is $g=0.9 \mathrm{~mm}$. When contact is effective, the sheet will start to experience bending until a forming angle of $90^{\circ}$ is achieved.

To let the sheet fully spring back, the forming phase is followed by the release of the tooling set-up and 


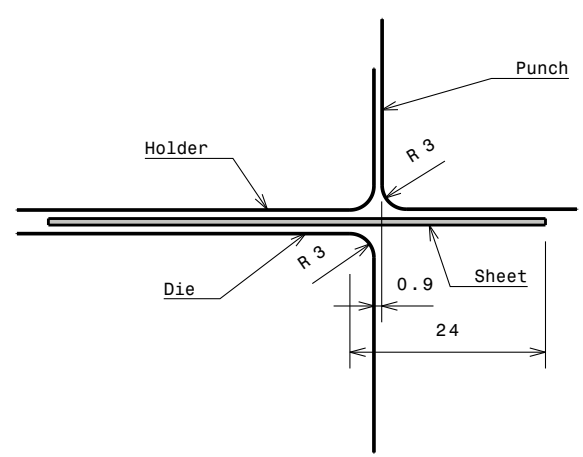

Figure 3: Lateral view of the geometrical tooling layout of the L-bending test

the death of the contact. All dimensions correspond to Case 1 in [4]. Results are compared to a mesh convergence study on Case 3 where $R_{d}=4 \mathrm{~mm}$ and $g=0.95 \mathrm{~mm}$. The sheet is divided into three different mesh areas resulting in $30+40+60 \times 8$ elements varying its density through thickness between 1 to 12 . This is made to have enough elements in contact with the die round geometry and improve accuracy in springback prediction. Our 8-node Q1/P0 brick mixed $u / p$ finite elements are used in the presented mesh in Fig. 4. Contact is modelled with an imposed constant friction coefficient of $\mu=0.125$. Tools are modelled as rigid surfaces and Materials \#104 and \#107 are employed in the simulations.
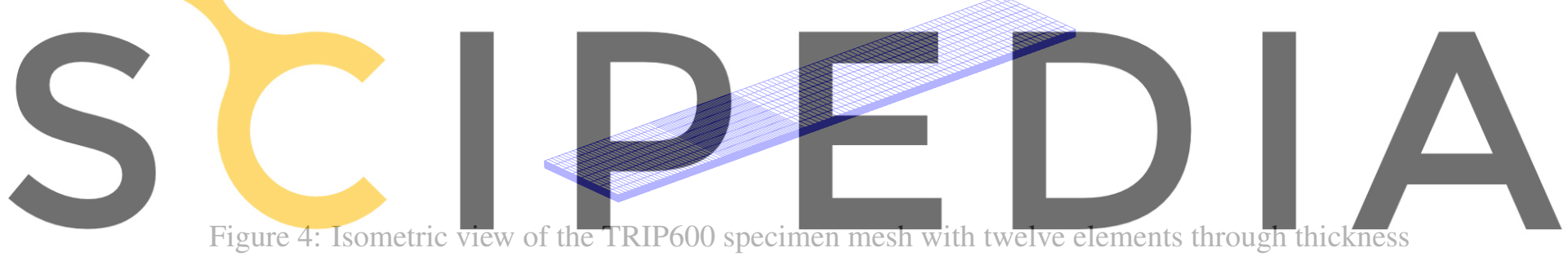

\section{Register for free at https//www.scipedia.com to download the version without the watermark In order to quantify the springback geometry, angle $\theta$ was defined in the after-springback geometry (Fig.}

5). Regarding sensitivity reasons, a mesh convergence study on Materials \#104 and \#107 was addressed, resulting in Fig. 6. Note that Chatti2011* and this work's convergences are related to different geometrical layouts which limits the validity of this comparison to mesh convergence. Great convergence is obtained, achieving successful springback predictions with three elements through thickness in comparison to the eight elements needed by Chatti2011.

Numerical values of the springback angle $\theta$ are presented in Table 3, re-validating the application of the material model to springback prediction. Note that only a combined hardening calibrated material manages to reproduce experimental results, while Material \#104 clearly underpredicts the springback angle $\theta$ as also proven in [4]. To finish the analysis of this benchmark, Fig. 7 presents the effective stress (Von Mises) of the deformed metallic sheets before and after springback has taken place.

\subsubsection{DRAW-BENDING TEST}

The draw-bending test is a well-known benchmark usually performed in sheet metal forming simulation literature. It basically consists of the study of a thin sheet metal strip subjected to a two-stage-bending 


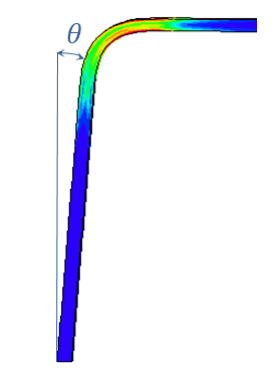

Figure 5: Definition of springback angle $\theta$

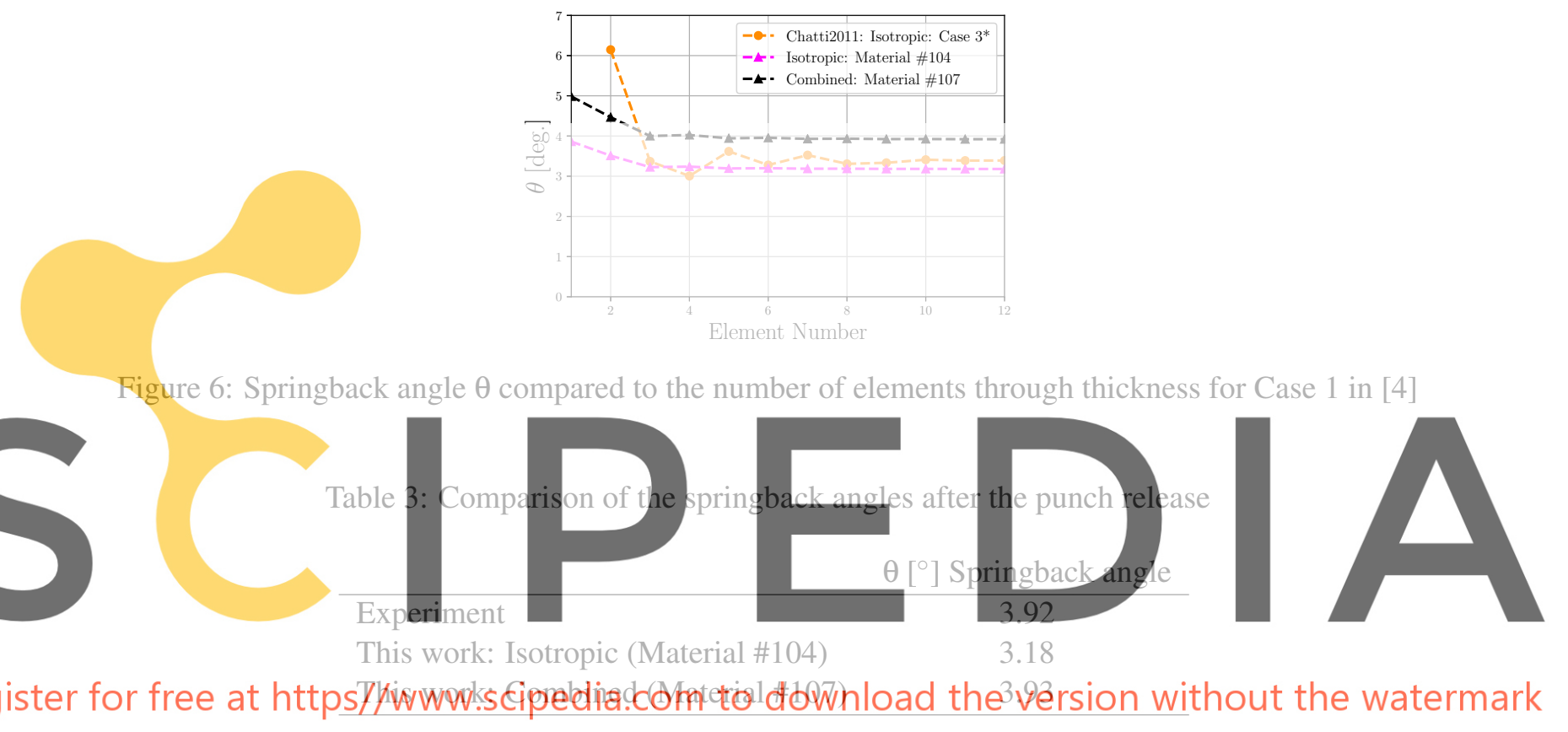

process following its hardening-sensitive springback. This framework makes this analysis very interesting in terms of springback prediction, applying the material model presented in the previous sections.

The machine involved in the experiments [5] has been reduced to two clamps jaws, in between the DP600 strip is placed, and a roller on which the strip is draw-bent. The tooling layout can be appreciated in Fig. 8 . The bending process starts with the pre-bending phase, where the strip is bent over the roller. The right clamping zone is controlled by displacement terms while the left clamp jaw is subjected to a tensile force. After complete pre-bending of the strip is achieved, the strip is drawn up to a prescribed displacement $\Delta x=167 \mathrm{~mm}$, following its unloading and release from the tooling. Afterwards, the specimen is allowed to fully spring back. The specimen initial dimensions are $430 \times 50 \times 1 \mathrm{~mm}$ and is modelled with our 8node Q1/P0 brick mixed $u / p$ elements $(200 \times 5 \times 1)$. A constant friction coefficient $\mu=0.02$ is selected as the roller is assumed to be a rotating cylindrical surface of $R=10 \mathrm{~mm}$ and $L=60 \mathrm{~mm}$. Fig. 9 presents the FE model before any loading is applied. The rest of test conditions are described in [5].

To measure the springback geometry, experimental data are compared to the simulation results. Fig. 10 shows the strip profiles at the end of the springback phase for Materials \#172, \#173 and \#174. It is 


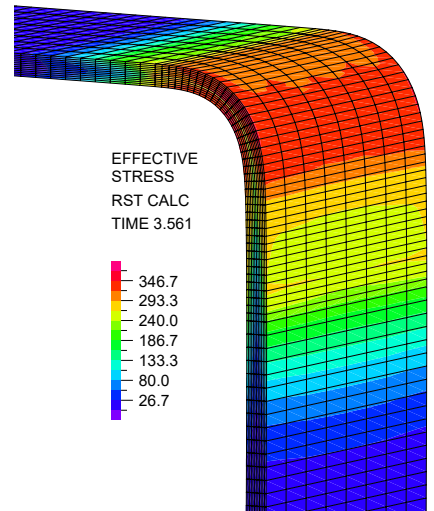

(a) Before springback

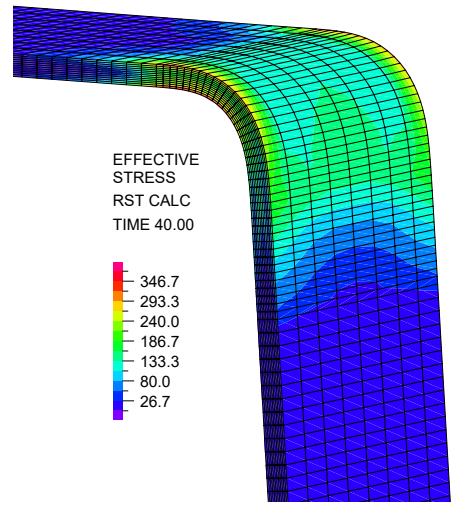

(b) After springback

Figure 7: Perspective view of the effective stress distribution in the L-Bending test for Material \#107

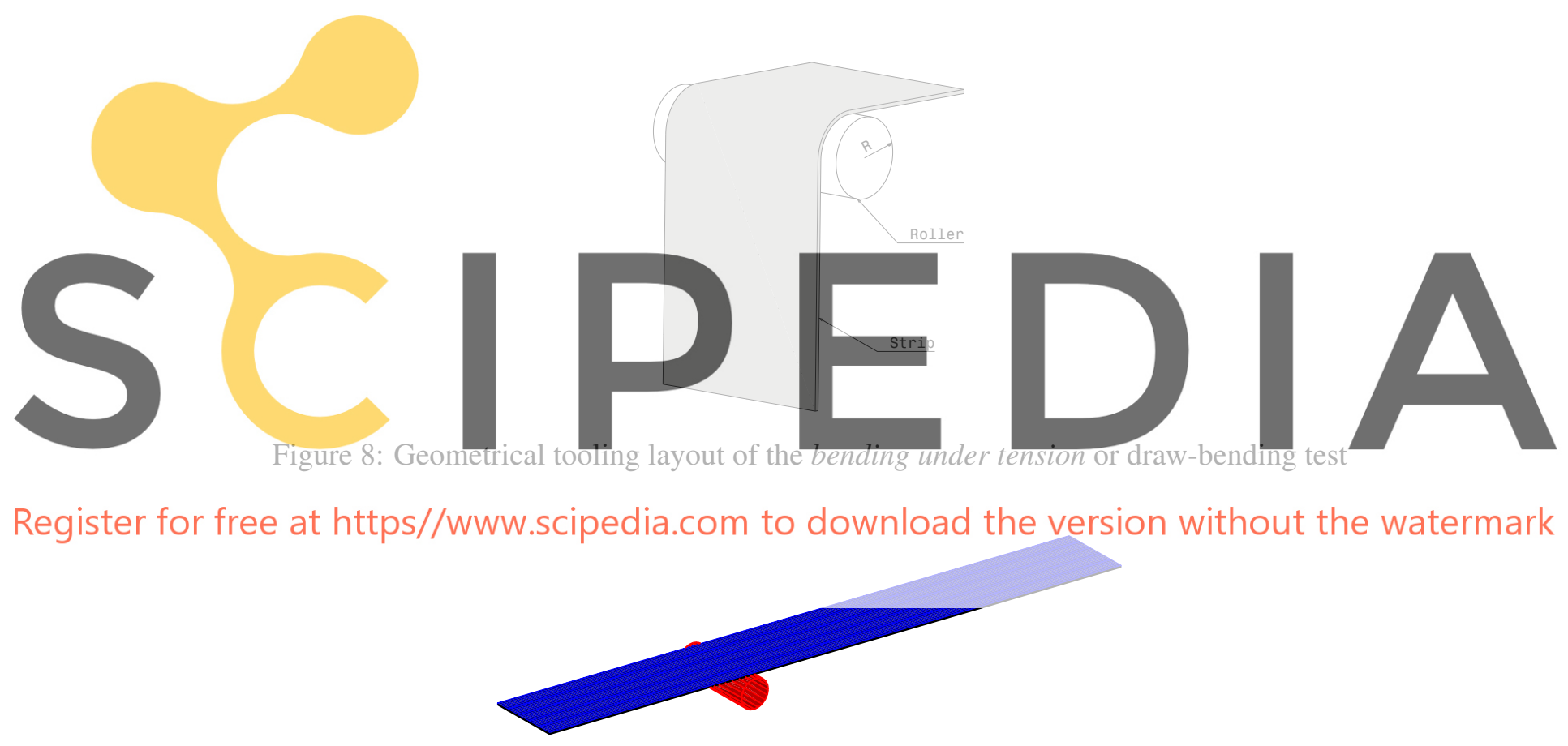

Figure 9: Perspective view of the FE model of the draw-bending test

trivial to deduce that an only-isotropic (NLIH) approach of the DP600 dual phase steel leads to a overprediction of the springback final geometry, while the use of an only-kinematic (LKH) material results in an underprediction by the same token. Only Material \#174 with combined hardening (NLIH+LKH), presents a successful prediction of springback for the experimental result, only differing at the very end of the profile. This result permits to re-validate the material model for the initial purpose of this work while this analysis outperforms the one presented in [7] where the imbalance resulted from an incomplete calibration lead to an imprecise prediction of springback. Nevertheless, note benchmark dimensions are 
dissimilar as the roller radius - strip thickness ratio is set in this work to $R / T=10$.

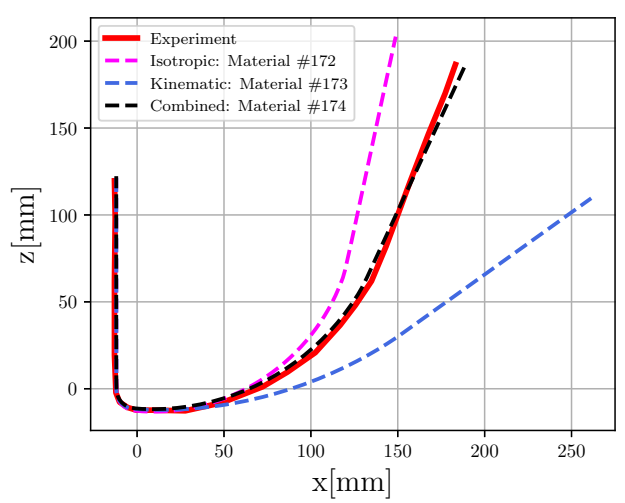

Figure 10: Experimental [5] and this work's strip profiles after the completion of the springback phase

\subsubsection{ACKNOWLEGMENTS}

Partial financial support for this work has been given by grant PGC 2018-097257-B-C32 from the Dirección General de Proyectos de Investigación of the Ministerio de Economía y Competitividad of Spain. The ADINA ${ }^{\circledR}$ program license used in this work has been a courtesy of ADINA R\&D to the UPM.

\section{REFERENCES}

[1] Latorre, M. and Montáns, F.J. A new class of plastic flow evolution equations for anisotropic multiplicative elastoplasticity based on the notion of a corrector elastic strain rate. Applied Mathematical Modelling, vol55, 716-740, (2018).

[2] Sanz M.A. and Montáns, F.J. and Latorre, M. Computational anisotropic hardening multiplicative elastoplasticity based on the corrector elastic logarithmic strain rate. Computer Methods in Applied Mechanics and Engineering, vol320, 82-121, (2017).

[3] Sanz M.A. and Nguyen K. and Rodríguez M. and Latorre M. and Montáns, F.J. Sheet metal forming analysis using a large strain anisotropic multiplicative plasticity formulation, based on elastic correctors, wich preserves the structure of the infinitesimal theory. Finite Elements in Analysis and Design, vol164, 1-17 (2019).

[4] Chatti, S. and Hermi, N. The effect of non-linear recovery on springback prediction. Computer and Structures, vol89, 1367-1377 (2011).

[5] Schwarze, M. and Vladimirov, I. N. and Reese, S. Sheet metal forming and springback simulation by means of a new reduced integration solid-shell finite element technology. Computer Methods in Applied Mechanics and Engineering, vol200, 454-476 (2011).

[6] Kojic, M. and Bathe, K.J. Inelastic analysis of solids and structures. Springer, pp 414 (2005).

[7] Díaz, R. and Sanz, M.A. and Nguyen, K. On modelling the finite strain anisotropic elastoplasticity with application to the prediction of springback in sheet metal forming. EUCASS: $8^{\text {th }}$ European Conference for Aeronautics and Aerospace Sciences (2019). 